\title{
Ultrasound guidance for pediatric vein cannulation: an emergency nurse quality improvement initiative and registry
}

\section{Domhnall O’Dochartaigh, Warren Ma, Christopher Picard, Richard Drew, Matthew J Douma, Tahira Daya, Sarah Curtis}

Background: Between 10 and 25 percent of pediatric patients present to the emergency department (ED) with difficult to cannulate veins. Recent RCT evidence suggests that in pediatric patients assessed at being a predicted difficult IV start (by DIVA score of 3 or more), ultrasound guided catheter placement decreased the number of IV attempts, decreased time to successful IV placement, and improved first pass success, patient satisfaction, and catheter dwell time. Our QI project examines the specific learnings around ultrasound guided peripheral IV in pediatric patients and suggests opportunity for non-pediatric specialist hospitals to consider with the overall aim of minimizing IV attempts on all pediatric patients within our EDs.

Building on a RCT led by Dr Curtis in pediatric patients conducted at the Stollery from 20122014, a standardized ultrasound guided nurse performed procedure was implemented in 2016 at the University of Alberta and Stollery EDs, and expanded to the Royal Alexandra ED in 2017 and the Misericordia ED in 2019. Using the same education package and QI study methodology as previously reported in adult patients this study focused specifically on pediatric patients.

Methods: A quality improvement (QI) registry was utilized to track complications and success of pediatric patients at all sites. The aim was to assess for program success, and improve education, training, and procedural success as required. Staff who had achieved independent practice voluntarily completed a tracking form whenever an ultrasound procedure occurred. Completed forms were assessed on a continual basis for any opportunities for improvement. Qualitative feedback was also obtained from informal interviews, a focus group, and a survey of the trained nurses. Feedback was thematically analyzed and grouped into themes for reporting.

Results: There were no reported pediatric UGIV placed at the MCH and RAH during the study period. At the Stollery 126 cases were reported. Immediate insertion complications were noted in three cases as 'pain or swelling at site', and 'unable to advance catheter'. In the first and second years of data collection the average number of traditional IV attempts prior to UGIV attempt decreased from 3.9 to 2.8; first ultrasound pass success increased from $65 \%$ to $86 \%$; overall ultrasound success improved from $85 \%$ to $97.6 \%$ respectively. Increasing nurse skill was significant with a linear increase of first pass and overall success seen with increasing number of ultrasound starts: From 6-20 starts (54\% first pass 64\% overall success) through to >150 starts (97\% first pass and 100\% overall). QI staff feedback included ensure adequate pediatric specific supplies such as longer length small gauge catheters, and a procedural focus of patient, provider, and assistant set up. Location of IV placement was noted to change in a number of cases from hand and $\mathrm{A} / \mathrm{C}$ to forearm.

\section{Advice and Lessons Learned:}


1) The key for staff to transition to procedural competance was to ensure initial and ongoing oportunities to place many ultrasound guided IVs (i.e. when time allows in all patients with non-optimal IV placement locations or with non-easy predicted tradititional IV starts)

2) Further work is required at non specilaist hospitals with trained staff to increase ultrasound guided use in pedatric patients

3) At all particapting sites work continues on unit level QI to minimize the number of IV attempts on all pedatric patients as well as work towards a cohort of available staff that are comforable and competent with ultrasound that can provide 24/7 unit coverage. (with limited numbers of trained staff there is increase burden on these staff to assist others while also completing their own nursing assignment. 